\title{
Waving plants and turbulent eddies
}

\section{J. J. F I N N I G A N}

CSIRO Marine and Atmospheric Research, Pye Laboratory, Black Mountain Laboratories, Canberra, ACT 2601, Australia

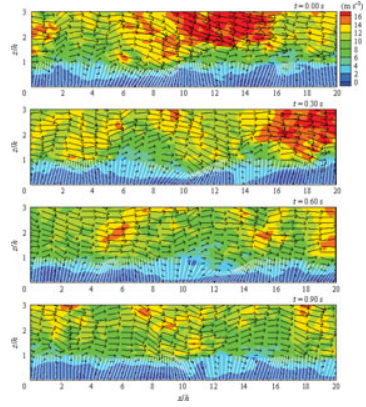

New large-eddy simulations of flow over a flexible plant canopy by Dupont et al. (J. Fluid Mech., 2010, this issue, vol. 652, pp. 5-44) have produced apparently paradoxical results. Work over the last three decades had suggested that turbulent eddies could 'lock onto' to the waving frequency of uniform cereal canopies. Their new simulations contradict this view, although a resolution may lie in the essentially three-dimensional nature of the instability process that generates the dominant eddies above plant canopies.

\section{Introduction}

The interaction between plants and fluid flow has been a perennial topic in fluid mechanics, biology and ecology (De Langre 2008). The most striking and studied example of plant-fluid interaction is 'honami', the regular marching waves seen in cereal crops on windy days. These crop waves are intimately connected with the transfer of momentum to the surface by turbulence, and Finnigan $(1979 a, b)$ was able to show that this transport was dominated by large coherent eddies with length scales of the order of the canopy height, $h$. His work coupled measurements of crop waving and of turbulent velocity and pressure to show the interdependence of turbulent structure and honami waves.

Py, De Langre \& Moulia (2006) concentrated on the interaction between the elastic stalks of the crop plants and the aerodynamic forcing to treat honami as a problem in aeroelasticity and fluid instability. They showed that some kind of resonant 'lock-in' occurred when the passage frequency of the dominant turbulent eddies approached the waving frequency of the stalks. Their conclusion has now been challenged by a much more complete simulation of a waving canopy by Dupont et al. (2010).

\section{Overview}

When honami occurs, a momentum gust or sweep bends over a group of plant stalks. (Here we assume right-handed Cartesian coordinates $(x, y, z)$ with $x$ in the streamwise and $z$ in the vertical direction. Corresponding velocity components are $\left(U+u^{\prime}, v^{\prime}, w^{\prime}\right)$, where capitals and primes denote appropriate time means and fluctuations.) As this sweep (with $u^{\prime}>0, w^{\prime}<0$ ) progresses downwind, the stalks spring back and perform several damped oscillations at their natural waving frequency, $f_{c}$, so that waves of crop deflection travel downwind, as shown in the figure beside the title from Dupont et al. (2010), which shows an $x-z$ cross-section of instantaneous wind-plant interaction at 
$0.3 \mathrm{~s}$ intervals. The colours represent the magnitude of the streamwise wind velocity, the arrows the wind vectors, and the white stems the plant displacements. Coherent waving occurs in patches (typically three or four times longer in the $x$ than in the $y$ direction) which contain about two or three wave crests. On windy days the whole crop is a superposition of these coherently waving patches (Finnigan 1979a; Py et al. 2005). The waving frequency $f_{c}$ is a function of the plants' elastic and inertial properties but the wavelength of the honami waves, $\lambda=U_{c} / f_{c}$ depends both on $f_{c}$ and on the sweep convection velocity $U_{c}$.

Some features of crops undergoing honami are generic. First, experiments agree that $U_{c}$ is significantly higher than $U(h)$, i.e. the mean windspeed at canopy top. Finnigan (1979a) found $U_{c}=1.8 U(h)$, while Dupont et al. (2010) find that $U_{c}=1.5 U(h)$. Second, the normalized root-mean-square pressure fluctuations measured at the soil surface below the canopy, $\sqrt{p^{\prime 2}} / \rho u_{*}^{2}$, where $p^{\prime}$ is the pressure fluctuation, $u_{*}$ is the friction velocity and $\rho$ is the air density, are typically three times larger than on a rough wall, a result that holds both in the field and for wind tunnels (e.g. Finnigan 1979a). Third, a key feature of a turbulent flow over a compliant surface with a distinct waving frequency, $f_{c}$ is that an independent time scale has been added to the scaling. In fully rough flows, velocity moments normalized with $u_{*}$ are independent of mean windspeed. In waving crops, however, such moments should also depend upon the reduced velocity, $U_{r}=U(h) /\left(f_{c} h\right)$.

The structure of turbulence in canopies and in the roughness sublayer, above the plants, is very different to that in the logarithmic surface layer. These differences are explained by the 'mixing-layer hypothesis' (Raupach, Finnigan \& Brunet 1996; Finnigan 2000). This hypothesis notes that the mean velocity profile has a characteristic inflexion point at the canopy top, such that the mean velocity $U(z)$ is inviscidly unstable. Therefore, it is hypothesized that a cascade of two-dimensional and three-dimensional instabilities results in large coherent eddies which are responsible for most of the turbulent transport and turbulent kinetic energy in these layers. As predicted by instability theory, eddy length scales are proportional to the vorticity thickness $\delta \equiv U(h) /[\mathrm{d} U / \mathrm{d} z(h)]$.

If ensemble means are taken, these coherent eddies take the form of superposed hairpin vortices with an underlying downstream 'head-up' (H-U) hairpin, in which the induced flow between the legs of the hairpin generates a momentum ejection $\left(u^{\prime}<0\right.$ and $w^{\prime}>0$ ) and an overlying upstream 'head-down' (H-D) hairpin, which generates a sweep between its legs. The convergence between the sweep and ejection produces a pulse of static pressure at $z=h$ (Finnigan et al. 2009). This eddy structure explains most observations of momentum and scalar transfer in uniform canopies.

The instability sequence begins with the initial (linear) inflection-point instability, a Kelvin-Helmholtz $(\mathrm{K}-\mathrm{H})$ mode with wavelength $\lambda$ proportional to $\delta$. The $\mathrm{K}-\mathrm{H}$ waves rapidly evolve (nonlinearly) into a train of transverse elliptical vortices, retaining the streamwise spacing, $\lambda$ of the initial linear instability. This vortex street is itself (linearly) unstable in the $y$ direction, and develops into a train of $\mathrm{H}-\mathrm{U}$ and H-D hairpin vortices. Finally, we observe (nonlinear) stretching and enhancement of hairpin vortex pairs by mean shear and self-induction (as shown in figure 1).

The growth rate of the two-dimensional and three-dimensional eigenmodes is proportional to $\mathrm{d} U / \mathrm{d} z(h)$, the mean shear at the canopy top, so that the eddies that dominate will be those that occur when $\mathrm{d} U / \mathrm{d} z(h)$ is elevated because a largescale gust from the boundary layer above has impinged on the canopy. Hence, the dominant eddies have downstream convection velocities substantially greater than $U(h)$. In plane mixing layers the two-dimensional instabilities can be influenced by 


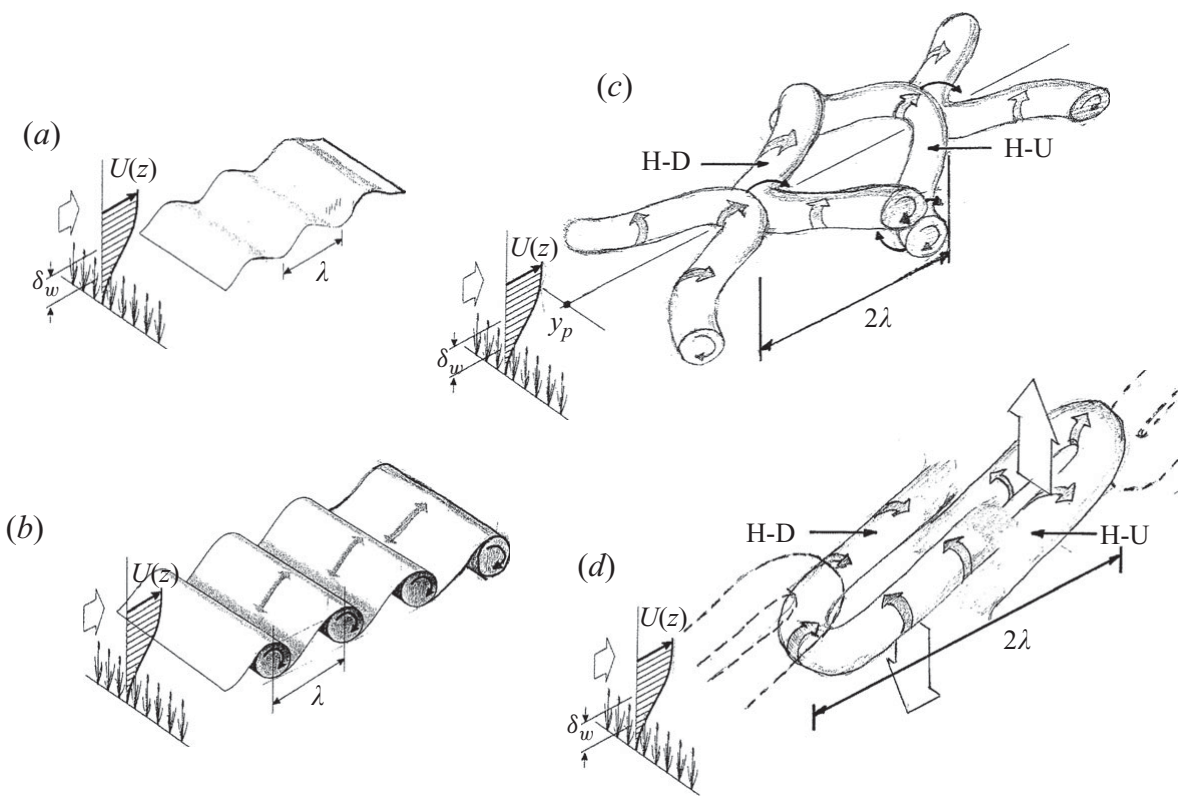

FIGURE 1. Schematic diagram of the dual-hairpin eddies formation (Finnigan, Shaw \& Patton 2009).

and 'lock onto' external excitation (Huerre \& Rossi 1998). The large coherent pressure fluctuations generated by honami waves, as well as the periodic drag of the waving stalks, seem to provide an ideal source of such excitation. Hence the results of Py et al. (2006), when they performed a stability analysis of the velocity field in a waving alfalfa crop, confirmed expectations based on both instability theory and experience. While their analysis was constrained to two dimensions, and concentrated on the initial two-dimensional K-H eigenmodes, they found a range of reduced velocity, where the two-dimensional instability locked on to the plant waving frequency and the 'eddy' growth rate was enhanced.

Dupont et al. (2010) extended this analysis by performing a full large-eddy simulation (LES) of an elastic canopy. They also improved the coupled instability model of Py et al. (2006) by replacing the piecewise linear profile of $U(z)$ by the LES computed profile and by including the effects of eddy viscosity. Their LES results reproduced observed honami behaviour in an alfalfa crop very well as well as reproducing the eddy structure predicted by the mixing-layer hypothesis. Their improved coupled instability model also reproduced the lock-in behaviour shown by Py et al. (2006). However, there were significant discrepancies between the results of the full LES simulations and their improved instability model. The LES results showed that the effect of the waving crop on the turbulent structure was negligible. No lock-in behaviour was observed.

\section{Future}

Dupont et al. (2010) suggest that this apparent paradox might result from a 'nonlinear saturation' mechanism which prevents the instability from displaying 
sensitivity to excitation by the waving plants. The results of Finnigan et al. (2009), described above, support this view because the energetic phase of the instability sequence is the three-dimensional hairpin pairing (figures $1 c$ and $1 d$ ). In fully developed mixing layers, the emergence of the three-dimensional form is rapid and the initial two-dimensional eigenmodes are unlikely to be observable in a canopy flow in a deep fully turbulent boundary layer.

It is possible that the three-dimensional hairpin pairs, once they have appeared, are insensitive to external forcing. Hence, the two-dimensional instability model may exaggerate the tendency to lock-in. Indeed, in deep fully developed diabatically neutral canopy flows, two-dimensional K-H modes have not been observed (although they are seen in shallow-water flows where the instability sequence does not progress to the three-dimensional stage, as discussed by Ghisalberti \& Nepf 2002). It is also important to realize that this phenomenological instability model only applies in the ensemble mean and should perhaps be regarded as an attractor towards which energetic eddies in the fully turbulent flow tend. Individual realizations are strongly distorted about this platonic ideal by the strong ambient turbulence so that any instability model in fully developed turbulence may exaggerate the sensitivity of the eigenmodes to external forcing. To test these ideas, the data of Dupont et al. (2010) could be analysed using the procedures in Finnigan et al. (2009) to see if three-dimensional hairpin vortex pairs dominate when the canopy is waving and, conversely, if shallow-water honami flow could be simulated to see whether lock-in occurs when the inflection point instability remains two-dimensional.

\section{References}

Brunet, Y., Finnigan, J. J. \& Raupach, M. R. 1994 A wind tunnel study of air flow in waving wheat: single-point velocity statistics. Bound.-Layer Meteorol. 70, 95-132.

De Langre, E. 2008 Effects of wind on plant. Annu. Rev. Fluid Mech. 40, 141-168.

Dupont, S., Gosselin, F., Py, C., De Langre, E., Hemon, P. \& Brunet, Y. 2010 Modelling waving crops using large-eddy simulation: comparison with experiments and a linear stability analysis. J. Fluid Mech. 652, 5-44.

FinNigan, J. J. 1979a Turbulence in waving wheat. I. Mean statistics and honami. Bound.-Layer Meteorol. 16, 181-211.

FinNigan, J. J. 1979b Turbulence in waving wheat. II. Structure of momentum transfer. Bound.-Layer Meteorol. 16, 213-236.

Finnigan, J. J. 2000 Turbulence in plant canopies. Annu. Rev. Fluid Mech. 32, 519-571.

Finnigan, J. J., Shaw, R. H. \& Patton, E. G. 2009 Turbulence structure above vegetation canopies. J. Fluid Mech. 637, 387-424.

Ghisalberti, M. \& NePF, H. 2002 Mixing layers and coherent structures in vegetated aquatic flow. J. Geophys. Res. 107, 1-11.

Huerre, P. \& Rossi, M. 1998 Hydrodynamic instabilities in open flow. In Hydrodynamics and Nonlinear Instabilities (ed. C. Godrèche \& P. Manneville), pp. 81-294. Cambridge University Press.

Py, C., De Langre, E. \& Moulia, B. 2006 A frequency lock-in mechanism in the interaction between wind and crop canopies. J. Fluid Mech. 568, 425-449.

Py, C., De Langre, E., Moulia, B. \& Hemon, P. 2005 Measurement of wind-induced motion of crop canopies from digital video images. Agric. Forest Met. 130, 223-236.

Raupach, M. R., Finnigan, J. J. \& Brunet, Y. 1996 Coherent eddies in vegetation canopies - the mixing layer analogy. Bound.-Layer Meteorol. 78, 351-382. 